\title{
In vitro plantlet regeneration from nodal segments and shoot tips of Capsicum chinense Jacq. cv. Naga King Chili
}

\author{
Mechuselie Kehie $\cdot$ Suman Kumaria $\cdot$ \\ Pramod Tandon
}

Received: 4 August 2011/Accepted: 6 September 2011/Published online: 27 September 2011

(C) The Author(s) 2011. This article is published with open access at Springerlink.com

\begin{abstract}
An in vitro regeneration protocol was developed for Capsicum chinense Jacq. cv. Naga King Chili, a very pungent chili cultivar and an important horticultural crop of Nagaland (Northeast India). Maximum number of shoot $(13 \pm 0.70)$ was induced with bud-forming capacity (BFC) index of 10.8, by culturing nodal segments in Murashige and Skoog (MS) medium supplemented with $18.16 \mu \mathrm{M}$ Thidiazuron (TDZ) followed by $35.52 \mu \mathrm{M}$ 6-benzylaminopurine (BAP). Using shoot tips as explants, multiple shoot $(10 \pm 0.37$ ) (BFC 8.3) was also induced in MS medium fortified with either $18.16 \mu \mathrm{M}$ TDZ or $35.52 \mu \mathrm{M}$ BAP. Elongated shoots were best rooted in MS medium containing $5.70 \mu \mathrm{M}$ indole-3-acetic acid (IAA). Rooted plantlets thus developed were hardened in 2-3 weeks time in plastic cups containing potting mixture of a 1:1 mix of soil and cow dung manure and then subsequently transferred to earthen pots. The regenerated plants did not show any variation in the morphology and growth as compared to the parent plant.
\end{abstract}

Keywords Capsicum chinense Jacq. · Nodal segments · Plant regeneration $\cdot$ Shoot tips

\section{Introduction}

Capsicum chinense Jacq. cv. Naga King Chili is an important spice crop of India belonging to the family Solanaceae. It is a self-pollinated species; however, the

M. Kehie · S. Kumaria $(\bowtie) \cdot$ P. Tandon Plant Biotechnology Laboratory, Department of Botany, Centre for Advanced Studies, North-Eastern Hill University, Shillong 793022, India

e-mail: sumankhatrikumaria@hotmail.com occurrence of high cross pollination leads to the formation of variants within Naga King Chili. C. chinense is a very pungent chili, measuring 1,001304 Scoville Heat Units (SHU). It is locally called as Naga King Chili (Bhoot jolokia or Naga jolokia in Assamese), and is native to North-Eastern India more particularly to Nagaland (Bhagowati and Changkija 2009).

In vitro plant regeneration from cells, tissues and organ cultures is a prerequisite for the application of plant biotechnology to plant propagation, plant breeding and genetic improvement. It is the only technology for the production of large quantities of "elite" planting material so as to increase the production and productivity. Micropropagation is advantageous over traditional propagation as it can be used to multiply novel plants, such as those that have been genetically modified or bred through conventional plant breeding methods. It is also used to provide a sufficient number of plantlets for planting from a stock plant which does not produce seeds or respond well to vegetative reproduction. It also leads to simultaneous accomplishment of rapid large-scale propagation of new genotypes (Tandon and Kumaria 2005). The conventional method of chili plant propagation using seeds is restricted by the short span of viability and low germination rate of seeds. Chili plants are also highly susceptible to fungal and viral pathogens (Morrison et al. 1986). Since chili plant lacks natural vegetative propagation, plant tissue culture technique provides an alternative method of propagating novel genotypes. The establishment of efficient and promising protocol for in vitro plantlet regeneration of Capsicum is required for the application of modern biotechnological tools, such as asexual reproduction of elite stocks, recovery of useful somaclonal variants, germplasm preservation as well as the production of transgenic plants with improved agronomic traits, interspecific hybrids, and haploid plants

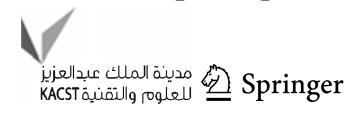


(Ezura 1997; Aguado-Santacruz et al. 2004). Though several attempts have been made on in vitro regeneration in the genus Capsicum, most of the reports are attempted on Capsicum annum (Agrawal et al. 1989; Ramirez-Malagon and Ochoa-Alejo 1996; Venkataiah et al. 2003; Khan et al. 2006) and a few on Capsicum frutescens (Subhash and Christopher 1988; Reddy et al. 2002). Only one report exists on in vitro propagation of $C$. chinense, wherein only six shoots per explant were obtained (Sanatombi and Sharma 2008). In this study, we present protocol for in vitro regeneration of $C$. chinense using both shoot tips and nodal segments resulting in higher number of shoots per cultured explants.

\section{Materials and methods}

C. chinense seeds (from dark red fruit) were obtained from a local field at Rüziephema village, Nagaland, India. The seeds were washed thoroughly in running tap water, then treated with $2 \%$ Labolene (v/v) for $10 \mathrm{~min}$ and finally rinsed five times with distilled water. These were then surface sterilized with $0.1 \% \mathrm{HgCl}_{2}$ (Himedia) for $5 \mathrm{~min}$ followed by several rinses with sterile distilled water. The sterilized seeds were inoculated on MS medium (Murashige and Skoog 1962) containing 3\% (w/v) sucrose and $0.8 \%(\mathrm{w} / \mathrm{v})$ agar, $\mathrm{pH}$ was adjusted to 5.8. Six-week-old in vitro germinated seedlings were used as the source of explants. Shoot tips $(1-2 \mathrm{~cm})$ and nodal segments $(1.0 \mathrm{~cm})$ were excised from these seedlings and implanted in the culture medium. The shoot bud induction medium was supplemented with various concentrations of either 6benzyl aminopurine, BAP (4.44-71.04 $\mu \mathrm{M})$ or Thidiazuron, TDZ (2.27-36.32 $\mu \mathrm{M})$. All media were autoclaved at $121{ }^{\circ} \mathrm{C}, 1.05 \mathrm{~kg} \mathrm{~cm}^{-2}$ pressure for $15 \mathrm{~min}$. Cultures were incubated at room temperature of $25 \pm 2{ }^{\circ} \mathrm{C}, 14 / 10-\mathrm{h}$ photoperiod with an irradiance of $62.2 \mu \mathrm{mol} \mathrm{m} \mathrm{m}^{-2} \mathrm{~s}^{-1}$ provided by cool white fluorescent tubes. The bud-forming capacity (BFC) was calculated based on the average number of buds and percentage of response of the explants (Tandon et al. 2007) as follows:

$\mathrm{BFC}=$ (average number of buds per explants $\times \%$ of explants forming buds $) \div 100$.

The effect of BAP and TDZ on BFC was evaluated by regression analysis. The elongated shoots $(1.5-2.5 \mathrm{~cm})$ were then excised and transferred to root induction medium containing various concentrations of either indole-3-acetic acid, IAA $(2.28-6.84 \mu \mathrm{M})$ or $\alpha$-naphthalene acetic acid, NAA $(0.53-4.29 \mu \mathrm{M})$. The number and length of the roots were recorded after 2 weeks of incubation. The well-rooted shoots were removed and washed thoroughly with distilled water. The plantlets were transplanted in perforated plastic cups filled with potting mixture of soil and cow dung (1:1) and irrigated with water daily in a green house. Finally, the hardened plants were transferred to earthen pots. All experiments were carried out with six replicates each and data were analyzed using one-way analysis of variance (ANOVA) at the 0.05 significance level in $\mathrm{JMP}^{\circledR}$ version 7.0.1 (SAS Institute, Cary, NC, USA).

The significant differences among the means were assessed by Tukey's Honestly Significant Difference (HSD) test used post-hoc on significant findings. Regression analysis for BFC index was performed using SPSS version 7.5 .

\section{Results and discussion}

Despite the economic importance of chili peppers, work on development of plant regeneration systems for Capsicum species has not progressed as in the case of several other solanaceous crops. In vitro regeneration of Capsicum species is reported to be difficult (Ochoa-alejo and Ramirezmalagon 2001).

In the present study, in vitro plantlet regeneration from shoot tips and nodal segments, cultured in MS medium supplemented with various concentrations of cytokinins (BAP or TDZ) alone are reported for $C$. chinense. TDZ is a phenyl urea that was originally developed as a cotton defoliant but has gained importance as a potent plant growth regulator for in vitro propagation systems of various crops (Fiola et al. 1990; Hutchinson et al. 1996). Based on review of available literature, very few reports exist on successful application of TDZ for rapid and efficient propagation of C. annuum (Venkataiah et al. 2003; Ahmad et al. 2006). Continuous exposure of explants to high concentration of TDZ is reported to be inhibitory/toxic, while low concentrations may not provide adequate stimulus (Huetterman and Preece 1993; Lu 1993; Hutchinson et al. 1996). In our study, the highest number of shoot buds $(13 \pm 0.70)$ with the BFC index of 10.8 was observed from nodal segments in MS medium containing 18.16 $\mu \mathrm{M}$ TDZ, followed by shoot tips ( $10 \pm 0.37)$ with BFC index of 8.33 (Figs. 1a, b, 3a, b). The effectiveness of low dose $(1.0 \mu \mathrm{M}$ TDZ) on multiple shoot induction in $C$. annuum has been reported earlier (Ahmad et al. 2006); however, in the present investigation with $C$. chinense, the frequency of shoot formation was more favorable at higher doses of TDZ. This may be due to the recalcitrant nature of this species. Earlier workers reported the use of very high concentration of BAP for maximal shoot proliferation from shoot tip explants of capsicum (Christopher and Rajam 1994; Sanatombi and Sharma 2008). However, in the present investigation with $35.52 \mu \mathrm{M}$ BAP alone, the 

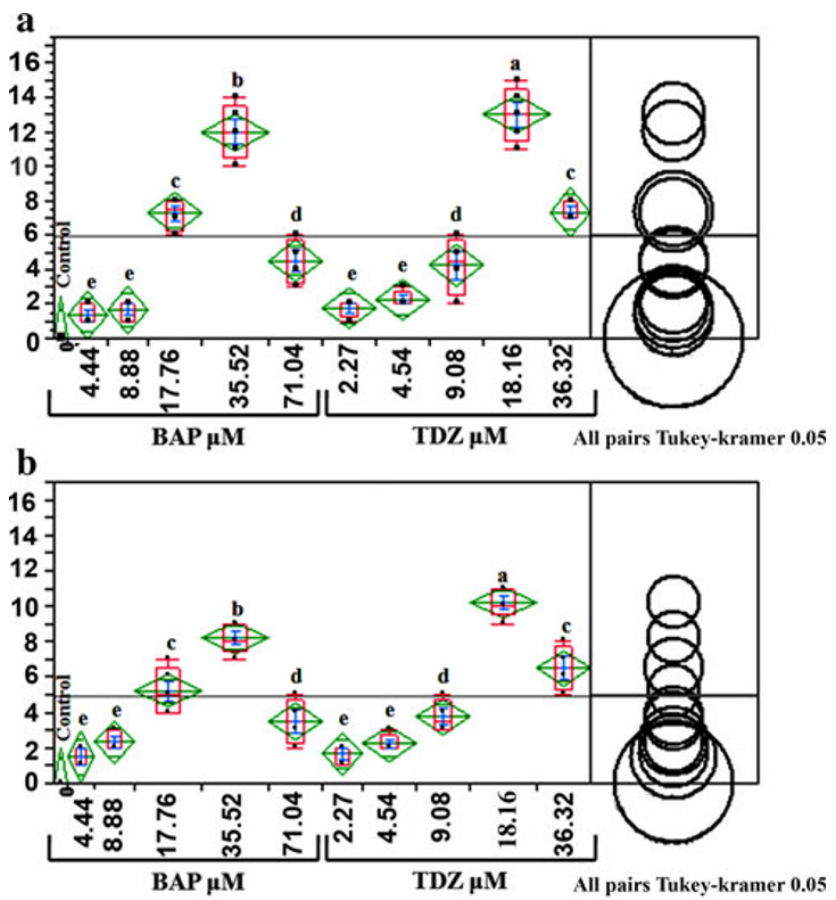

Fig. 1 Effect of 6-benzylaminopurine and thidiazuron on shoot induction from nodal segments (a) and shoot tips (b) of Capsicum chinense Jacq. cv. Naga King Chili. in MS medium. Data scored after 5 weeks of culture. Tukey's post-hoc test shows means that are not significantly different grouped by the same letter

maximum number of shoot induced from nodal segments was $12 \pm 0.70$ with BFC index of 7.99, followed by shoot tips $(8.2 \pm 0.37)$ with BFC index of 6.83. This increase in shoot formation is nearly twofold more than what has been reported earlier by Sanatombi and Sharma (2008). The BFC index is an efficient indicator of bud induction as it takes into consideration both the number of explants showing bud induction as well as the number of buds per explants (Tandon et al. 2007). An analysis of regression showed that regardless of the explants types used (nodal segment and shoot tip), the BFC gradually decreased at a concentration higher than $35.52 \mu \mathrm{M}$ BAP. Whereas, in the case of TDZ as growth regulator, the most suitable TDZ level $(18.16 \mu \mathrm{M}$ TDZ) for $C$. chinense produced $\mathrm{BFC}$ index of 10.8 shoots per explant. Elevation of TDZ level beyond this concentration drastically reduced BFC index as indicated by regression analysis (Fig. 2a-d). This may be due to the toxic effect of TDZ at higher doses.

Unlike other solanaceous species, chili has been a recalcitrant species with regard to its capacity for in vitro plant regeneration (Liu et al. 1990). The rate of progress in Capsicum is relatively slower than other members of solanaceae because of its high genotypic dependence and recalcitrant nature. Capsicum is a recalcitrant plant in terms of in vitro cell, tissue and organ differentiation (Kothari et al. 2010). Direct organogenesis has been the most frequently used morphogenic route for in vitro chili plant regeneration. However, the major problem faced to achieve this goal has been the failure of elongation of the induced shoot buds (Ochoa-alejo and Ramirez-malagon 2001). Because of ill-defined buds, leafy or shoot like structures which fail to elongate, several attempts have been made by various workers to enhance elongation of shoot buds (Frank-Duchenne et al. 1998; Venkataiah et al. 2003; Sanatombi and Sharma 2008). In our study, maximum elongation of shoots $(1.5-2.5 \mathrm{~cm})$ was observed in MS Medium containing $18.16 \mu \mathrm{M}$ TDZ or $35.52 \mu \mathrm{M}$ BAP (Fig. 3c).

The elongated shoots were excised individually and cultured in MS medium containing either IAA $(2.28-6.8 \mu \mathrm{M})$ or NAA $(0.53-4.29 \mu \mathrm{M})$ for root induction. The regenerated shoots rooted within 2 weeks of treatment but with different rooting frequency in each treatment. The effects of auxins on root induction in in vitro regenerated chili plantlets have been reported (Agrawal et al. 1989; Christopher and Rajam 1996). In the present investigation, the chili plantlets were best rooted in a medium containing $5.70 \mu \mathrm{M}$ IAA, with the highest number $(28.4 \pm 0.50)$ and maximum length $(4.18 \pm 0.26 \mathrm{~cm})$ of roots (Table 1; Fig. 3d). Our observations are consistent with the earlier finding in which IAA was successfully employed for rooting in C. chinense (Sanatombi and Sharma 2008). Among the two auxins tested, IAA resulted in more effective root induction as compared to NAA in which the roots were short and thick. The rooted plantlets after 2-3 weeks were transferred to potting mixture of a 1:1 mix of soil and cow dung and kept in green house for hardening and acclimatization (Fig. 3e), where they showed 90\% survival. The regenerated plants did not show any variation in the morphology and growth as compared to the parent plant. The hardened plantlets thus were transplanted and established in earthen pots and bore normal fruits (Fig. 3f, g).

\section{Conclusion}

The present study demonstrates a simple and promising protocol for in vitro plantlet regeneration of $C$. chinense from nodal segments and shoots tips which is nearly twofold more than what has been reported earlier. The use of either BAP or TDZ favored the development of plant. However, the use of TDZ at a concentration higher than $18.16 \mu \mathrm{M}$ TDZ appeared to be toxic. IAA may be preferred over NAA for root induction of this species. This protocol may be applied for conservation and large-scale propagation of individual genotypes of this species of chili. 
Fig. 2 Effect of either BAP $(4.44,8.88,17.76,35.52$ and $71.04 \mu \mathrm{M})$ or TDZ $(2.27,4.54$, $9.08,18.16$ and $36.32 \mu \mathrm{M})$ concentration on bud-forming capacity from $C$. chinense shoot tip $(\mathbf{a}, \mathbf{b})$ and nodal explants (c, d), after 5 weeks of culture
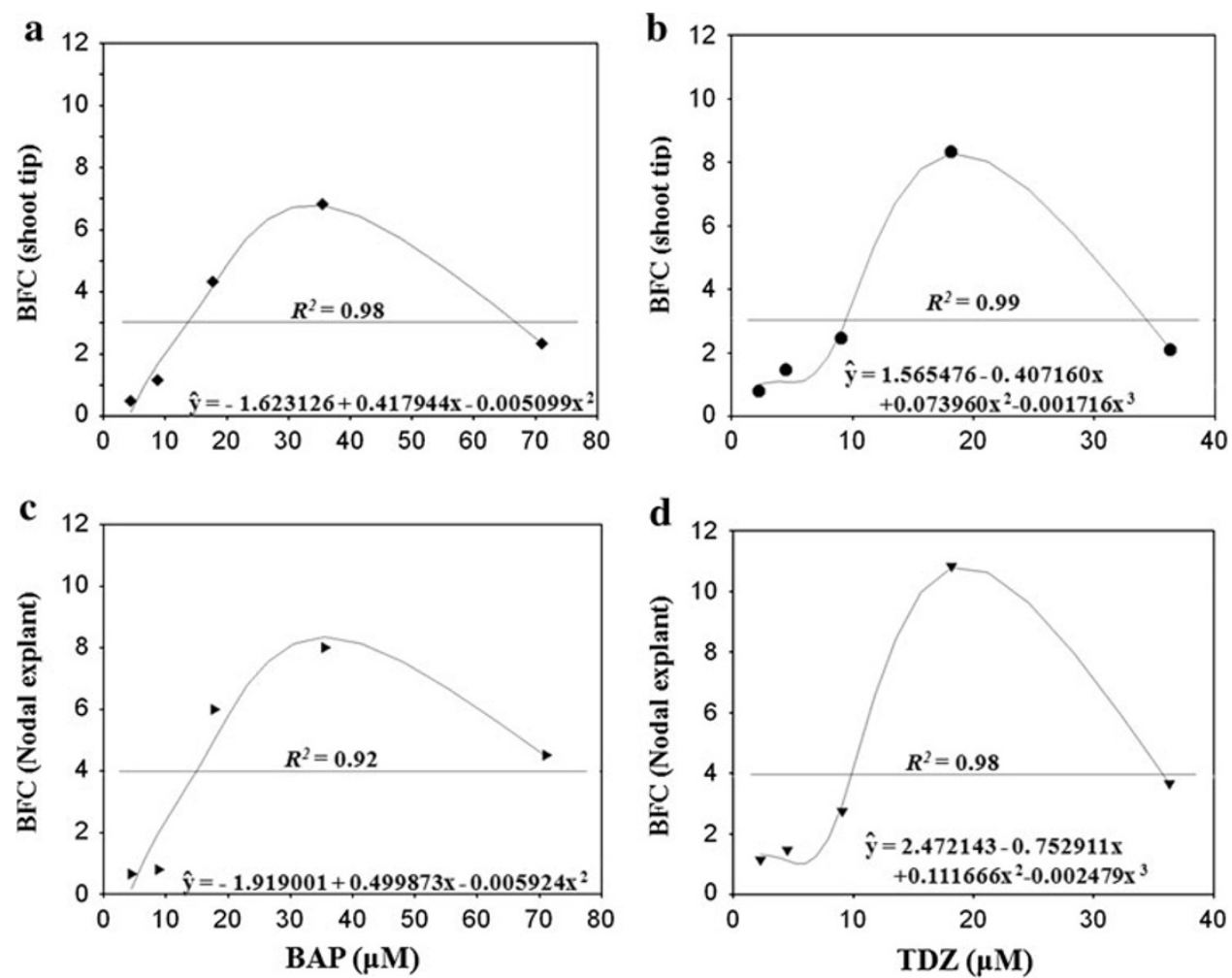
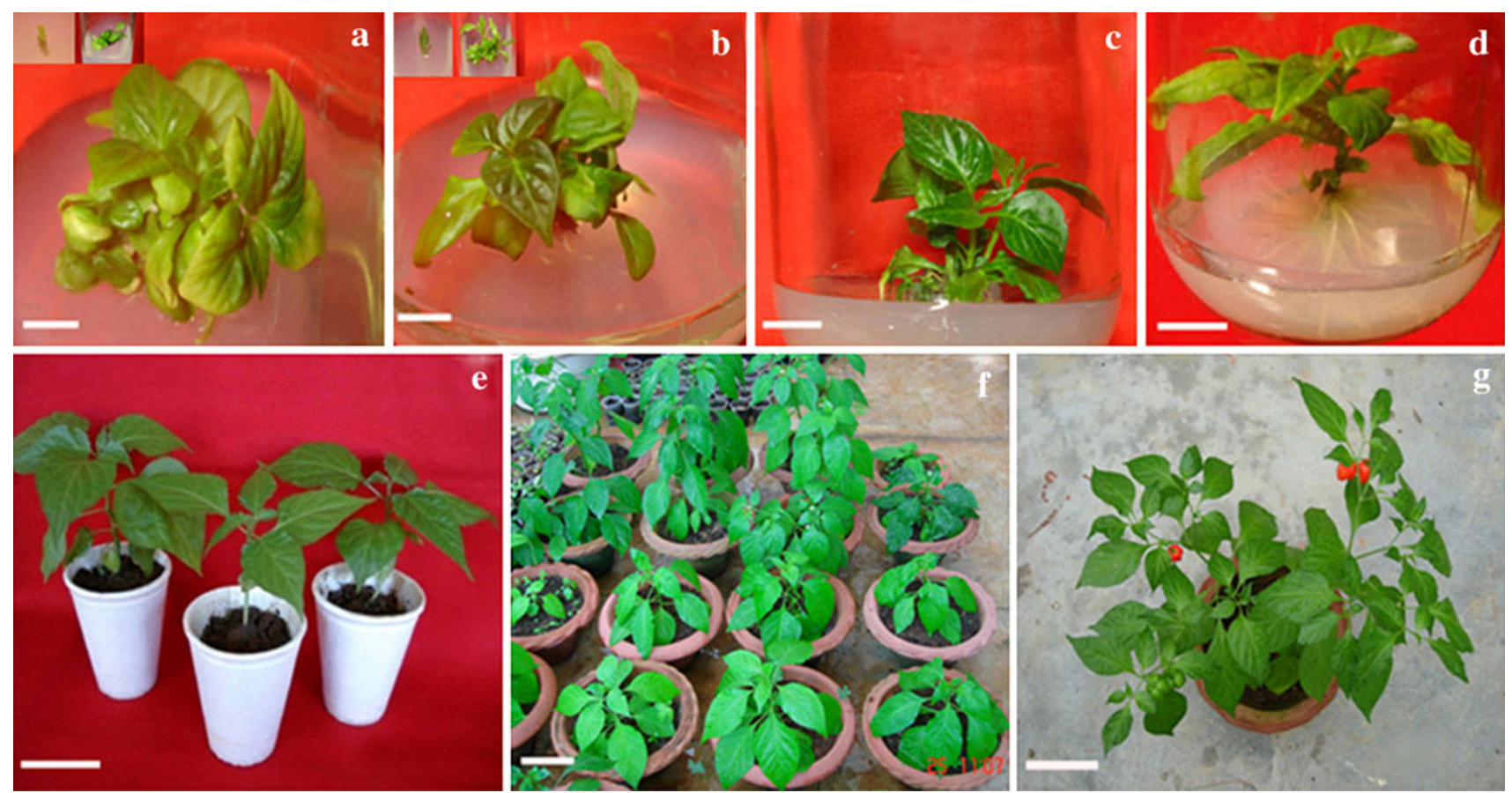

Fig. 3 In vitro regeneration in Capsicum chinense Jacq. cv. Naga King Chili. a Shoot induction from nodal explants in MS $+18.16 \mu \mathrm{M}$ TDZ after 5 weeks $($ Bar $1 \mathrm{~cm})$. b Shoot induction from shoot tip explants in MS $+18.16 \mu \mathrm{M}$ TDZ after 5 weeks (Bar $1 \mathrm{~cm}$ ). c Elongated plantlets of regenerated shoots $($ Bar $1 \mathrm{~cm})$. d Rooting of in vitro regenerated shoots after 3 weeks of culture $(B a r 1 \mathrm{~cm})$. e Regenerated hardened plantlets $($ Bar $3 \mathrm{~cm})$. f Regenerated plantlet transferred to earthen pot (Bar $4 \mathrm{~cm})$. $\mathbf{g}$ Regenerated plantlets bearing healthy fruit 
Table 1 Effect of auxins on rooting of shoots from shoot tips and nodal segments of $C$. chinense in MS medium

\begin{tabular}{lllll}
\hline Auxins & $\begin{array}{l}\text { Concentration } \\
(\mu \mathrm{M})\end{array}$ & $\begin{array}{l}\text { Number of } \\
\text { roots }\end{array}$ & $\begin{array}{l}\text { Length of roots } \\
(\mathrm{cm})\end{array}$ & $\begin{array}{l}\text { Response } \\
(\%)\end{array}$ \\
\hline Control & - & - & - & - \\
IAA & 2.28 & $14.0 \pm 0.70 \mathrm{c}$ & $1.5 \pm 0.22 \mathrm{c}$ & 100 \\
& 3.42 & $17.4 \pm 0.67 \mathrm{~b}$ & $1.6 \pm 0.18 \mathrm{c}$ & 100 \\
& 4.56 & $18.2 \pm 1.06 \mathrm{~b}$ & $2.4 \pm 0.24 \mathrm{~b}$ & 100 \\
& 5.70 & $28.4 \pm 0.50 \mathrm{a}$ & $4.1 \pm 0.26 \mathrm{a}$ & 100 \\
& 6.8 & $12.8 \pm 0.86 \mathrm{~cd}$ & $3.7 \pm 0.30 \mathrm{a}$ & 100 \\
NAA & 0.53 & $10.0 \pm 0.70 \mathrm{de}$ & $0.6 \pm 0.05 \mathrm{~d}$ & 100 \\
& 1.07 & $10.2 \pm 0.86 \mathrm{de}$ & $0.5 \pm 0.12 \mathrm{~d}$ & 100 \\
& 2.14 & $14.0 \pm 0.70 \mathrm{c}$ & $0.6 \pm 0.12 \mathrm{~d}$ & 100 \\
& 3.22 & $10.2 \pm 0.58 \mathrm{de}$ & $0.2 \pm 0.03 \mathrm{~d}$ & 90 \\
& 4.29 & $8.2 \pm 0.58 \mathrm{e}$ & $0.1 \pm 0.02 \mathrm{~d}$ & 80 \\
\hline
\end{tabular}

Data scored after 2 weeks of culture. Means + SE. Means followed by the same letters are not significantly different according to Tukey's HSD

Acknowledgments Mechuselie Kehie would like to acknowledge the University Grant Commission (UGC) for awarding him Research Fellow for Meritorious students.

Open Access This article is distributed under the terms of the Creative Commons Attribution License which permits any use, distribution and reproduction in any medium, provided the original author(s) and source are credited.

\section{References}

Agrawal S, Chandra N, Kothari SL (1989) Plant regeneration in tissue cultures of pepper (Capsicum annum L cv. Mathania). Plant Cell Tissue Organ Cult 16:47-55

Aguado-Santacruz GA, Martínez-Castillo A, Moreno-Gómez B, Peña-Mosqueda D (2004) Cultivo de tejidos vegetales. In: Aguado-Santacruz GA (ed) XVI Jornada de ingeniería bioquímica. INIFAP, Unidad de Biotecnología-Instituto Tecnológico de Celaya, Celaya, Gto. Mexico

Ahmad N, Siddique I, Anis M (2006) Improved plant regeneration in Capsicum annum L From nodal segments. Biologia Plantarum 50:701-704

Bhagowati RR, Changkija S (2009) Genetic variability and traditional practices in Naga King Chili landraces of Nagaland. Asian AgriHistory 13:171-180

Christopher T, Rajam MV (1994) In vitro clonal propagation of Capsicum spp. Plant Cell Tissue Organ Cult 38:25-29

Christopher T, Rajam MV (1996) Effect of genotype, explant and medium on in vitro regeneration of red pepper. Plant Cell Tissue Organ Cult 46:245-250

Ezura H (1997) Micropropagation of Capsicum species (pepper). In: Bajaj YPS (ed) Biotechnology in agriculture and forestry high- tech and micropropagation, vol 39. Springer, Heidelberg, pp 48-59

Fiola JA, Hassan MA, Swartz HJ, Bors RH, Mcnicol R (1990) Effect of thidiazuron, light fluency rates and kanamycin on in vitro shoot organogenesis from excised rubus cotyledons and leaves. Plant Cell Tissue Organ Cult 20:223-228

Frank-Duchenne M, Wang Y, Ben Tahar S, Beachy RN (1998) In vitro stem elongation of sweet pepper in media containing 24-epi-brassinolide. Plant Cell Tissue Organ Cult 53:79-84

Huetterman CA, Preece JE (1993) Thidiazuron: a potent cytokinin for woody plant tissue culture. Plant Cell Tissue Organ Cult 33:105-119

Hutchinson MJ, Murch SJ, Saxena PK (1996) Morphoregulatory role of thidazuron: evidence of the involvement of endogenous auxin in thidiazuron-induced somatic embryogenesis of geranium (Perlargonium $x$ hortorum Baile). J Plant Physiol 149:573-579

Khan H, Siddique I, Anis M (2006) Thidiazuron induced somatic embryogenesis and plant regeneration in Capsicum annum. Biologia Plantarum 50:789-792

Kothari SL, Joshi A, Kachhwaha S, Ochoa-Alejo N (2010) A review on tissue culture and transgenesis. Biotechnol Adv 28:35-48

Liu W, Parrott WA, Hildebrand DF, Cillins GB, Williams EG (1990) Agrobacterium induced gall formation in bell pepper (Capsicum annum L.) and formation of shoot like structure expressing introduced genes. Plant Cell Rep 9:360-364

Lu CY (1993) The use of Thidiazuron in tissue culture. In vitro cellular development biology. Plant 29:92-96

Morrison RA, Kining RE, Evans DA (1986) Pepper. In: Evans DA, Sharp WR, Ammirato PV (eds) Handbook of plant cell culture, vol 4. McGraw Hill, New York, pp 554-573

Murashige T, Skoog F (1962) A revised medium for rapid growth and bioassays with tobacco tissue cultures. Physiol Plant 15:473-497

Ochoa-alejo N, Ramirez-malagon R (2001) In vitro chili pepper biotechnology. In vitro cellular \& developmental biology. Plant 37:701-729

Ramirez-Malagon R, Ochoa-Alejo N (1996) An improved and reliable chili pepper (Capsicum annum L.) plant regeneration method. Plant Cell Rep 16:226-231

Reddy OB, Giridhar P, Ravishankar GA (2002) The effect of tricontanol on micropropagation of Capsicum frutescens and Decalepis hamiltonii W\&A. Plant Cell Tissue Organ Cult 71:253-258

Sanatombi K, Sharma GJ (2008) In vitro propagation of Capsicum chinense Jacq. Biologia Plantarium 52:517-520

Subhash K, Christopher T (1988) Direct plantlet formation in cotyledon cultures of Capsicum frutscens. Curr Sci 57:99-100

Tandon P, Kumaria S (2005) Prospects of Plant Conservation Biotechnology in India with special reference to Northeastern Region. In: Tandon P, Sharma M, Swarup R (eds) Biodiversity: status and prospects. Narosa Publishing House, New Delhi, pp 79-92

Tandon P, Kumaria S, Choudhury H (2007) Plantlet regeneration of Pinus kesiya Royle ex Gord. From mature embryos. Indian J Biotechnol 6:262-266

Venkataiah P, Christopher T, Subhash K (2003) Thidiauron induced high frequency adventitious shoot formation and plant regeneration in Capsicum annum L. Plant Biotechnol 5:245-250 